\title{
DEGRADACIÓN DE LAS ENZIMAS FIBROLÍTICAS DE TRAMETES SP. EUM1, PLEUROTUS OSTREATUS IE8 Y DE FIBROZYME®
}

\author{
DEGRADATION OF FIBROLYTIC ENZYMES FROM TRAMETES SP. EUM1, \\ PLEUROTUS OSTREATUS IE8 AND FIBROZYME®
}

\author{
Márquez Araque, A.T. ${ }^{1}$, Mendoza Martínez, G.D. ${ }^{2 *}$, González Muñoz, S.S. ${ }^{3}$, Buntinx Dios, S. ${ }^{4}$ \\ Meneses Mayo, M. ${ }^{3}$ y Loera Corral, O. $^{5}$
}

\begin{abstract}
${ }^{1}$ Decanato de Ciencias Veterinarias. Universidad Centroccidental Lisandro Alvarado. Núcleo Dr. Hector Ochoa Zuleta. Cabudare. CP 3011. Edo. Lara. Venezuela.

${ }^{2}$ Universidad Autónoma Metropolitana. Xochimilco. Calz. del hueso 1100. CP 04960. D.F. México. *gmendoza@correo.xoc.uam.mx

${ }^{3}$ Colegio de Postgraduados, Montecillo. Carretera México-Texcoco km. 36.5. CP 09470. Estado de México. México.

${ }^{4}$ Universidad Nacional Autónoma de México. Circuito Exterior. Ciudad Universitaria. CP 04510. D.F. México. ${ }^{5}$ Universidad Autónoma Metropolitana. Iztapalapa. Av. San Rafael Atlixco 186. CP 09340. D.F. México.
\end{abstract}

\section{Palabras clave adicionales}

Hongos. Sustrato. N amoniacal.

\section{RESUMEN}

Se determinó la degradación en líquido ruminal de las enzimas producidas por los hongos Trametes sp. EUM1, P. ostreatus IE8 y de las presentes en un producto fibrolítico comercial (Fibrozyme ${ }^{\circledR}$ ) mediante la medición del nitrógeno amoniacal $\left(\mathrm{N}-\mathrm{NH}_{3}\right)$ liberado. Para cada enzima los resultados se analizaron como un experimento factorial $(3 \times 2 \times 2)$ con los factores: sustrato (xilano, carboximeticelulosa y sin sustrato), nivel de proteína de la enzima (1 ó 2) y el tiempo de contacto de la enzima con el sustrato previo a la incubación (0 ó 1 h). La concentración de $\mathrm{N}-\mathrm{NH}_{3}$ en líquido ruminal fue diferente $(p \leq 0,05)$ entre sustratos en todos las enzimas estudiadas. El nivel de proteína de la enzima tuvo efecto $(p \leq 0,05)$ sobre la concentración de $\mathrm{N}-\mathrm{NH}_{3}$ en el líquido ruminal con enzimas de Trametes sp. y $P$. ostreatus, pero no en el incubado con Fibrozyme. La interacción sustrato por nivel de proteína tuvo efecto $(p \leq 0,05)$ en la concentración de $\mathrm{N}-\mathrm{NH}_{3}$ en las enzimas de Trametes sp. y $P$. ostreatus. Se observaron menores concentraciones de $\mathrm{N}-\mathrm{NH}_{3}$ al incubar las enzimas con un sustrato, por lo que se considera un factor importante en la estabilidad de las enzimas estudiadas.

Recibido: 11-3-07. Aceptado: 1-10-07.

\section{AdDitional KeYWords}

Fungi. Substrate. Ammoniacal N.

\section{SUMMARY}

Ruminal degradation of enzymes produced by fungi Trametes sp. EUM1, Pleurotus ostreatus IE8, and from a commercial product (Fibrozyme ${ }^{\circledR}$ ) was determined trough ammonia nitrogen $\left(\mathrm{N}-\mathrm{NH}_{3}\right)$ released. For each enzyme results were analyzed as a factorial experiment $(3 \times 2 \times 2)$; factors were: substrate (xylan, carboximethycellulose and no substrate), protein level of enzyme (1 or 2) and times of contact enzyme-substrate previous incubation ( 0 or $1 \mathrm{~h}$ ). The $\mathrm{NH}_{3}-\mathrm{N}$ concentration in ruminal fluid was different $(p \leq 0.05)$ between substrates on all studied enzymes. The protein level of enzyme had effect $(p \leq 0.05)$ on $\mathrm{NH}_{3}-\mathrm{N}$ concentration from Trametes sp. or $P$. ostreatus enzymes, but not from Fibrozyme. The substrate by protein level had effect $(p \leq 0.05)$ in the $\mathrm{NH}_{3}-\mathrm{N}$ concentration in the Trametes sp. and $P$. ostreatus enzymes. The time of contact enzyme-substrate effect was not significant for any of studied enzymes. Because minor $\mathrm{N}-\mathrm{NH}_{3}$ concentration was observed when enzymes were incubated with a substrate, this indicates that degradation of enzymes in ruminal liquid was reduced in presence of a substrate; therefore substrate is important in the stability of studied enzymes.

Arch. Zootec. 59 (225): 145-148. 2010. 


\section{INTRODUCCIÓN}

Una estrategia para mejorar la utilización de los alimentos y, en particular, de materiales fibrosos es el uso de enzimas fibrolíticas exógenas para el tratamiento de forraje o incluidas como suplemento en la ración (Dawson y Tricarico, 1999). En el rumen, las enzimas podrían ser degradadas por las proteasas microbianas. Sin embargo, algunos estudios indican que las enzimas parecen ser estables en el rumen (Hristov et al., 1998; Morgavi et al., 2001) y complementar la actividad fibrolítica microbiana ruminal o incrementarla (Morgavi et al., 2000). La formación del complejo enzima-sustrato puede ser importante en la estabilidad de las enzimas, en el líquido ruminal y el contacto previo de la enzima con el sustrato aparentemente favorece el efecto benéfico de la enzima (Fontes et al., 1995; McAllister et al., 1999). Los hongos Trametes sp. EUM1 y $P$. ostreatus IE8 producen enzimas fibrolíticas, las cuales podrían ser utilizadas como aditivos en la alimentación de los rumiantes, por ello se planteó como objetivo evaluar el efecto del sustrato, el nivel de proteína y el tiempo de contacto de la enzima con el sustrato sobre la degradación en el líquido ruminal de las enzimas producidas por estos hongos y de las presentes en un producto comercial.

\section{MATERIAL Y MÉTODOS}

Las enzimas de Trametes sp. EUM1 y $P$. ostreatus IE8 se produjeron por fermentación sólida en bagazo de caña de azúcar. La extracción de las enzimas se realizó con una solución amortiguadora de citrato, $50 \mathrm{Mmol}$, y a pH 5,3. El producto comercial (Fibrozyme, Alltech) se preparó a razón de $1 \mathrm{~g} / 100 \mathrm{ml}$ de agua destilada. La concentración de proteína de los extractos se determinó por el método de Bradford (1976). Los niveles de proteína (NP) fueron diferentes en cada enzima: Trametes sp.: nivel $1=0,28$ y nivel $2=$ $0,55 \mathrm{mg}$; $P$. ostreatus: nivel $1=0,14$ y nivel $2=$ 0,28 mg y Fibrozyme: nivel 1=0,19 y nivel $2=$
$0,38 \mathrm{mg}$. Las enzimas se aplicaron en forma líquida a razón de 1 y 2 ml para los NP 1 y 2 . Los sustratos (xilano de avena; SigmaAldrich X0627 y carboximetilcelulosa sal de sodio,CMC; Sigma-Aldrich C4888) se incubaron con las enzimas por triplicado en $20 \mathrm{ml}$ de líquido ruminal mezclado con saliva artificial de McDougall's (1:4 v/v), durante 3, 6 y 12 horas. La concentración de amoniaco como indicador de la degradación de la proteína en el líquido ruminal se midió utilizando el método de Indophenol (McCulloug, 1967). Cada enzima se analizó como un experimento factorial $(3 \times 2 \times 2)$. Los factores fueron: el sustrato (xilano, CMC y sin sustrato), el NP del extracto enzimático (1 ó 2) y los tiempos de contacto entre el sustrato con la enzima ( 0 ó 1 h). Los datos se analizaron con el PROC GLM (SAS, 2001). Los promedios de la concentración de $\mathrm{N}-\mathrm{NH}_{3}$ se compararon mediante la prueba de Tukey (Steel y Torrie, 1986).

\section{RESULTADOSYDISCUSIÓN}

Para la enzima de Trametes sp. sin sustrato la concentración de $\mathrm{N}-\mathrm{NH}_{3}$ fue mayor $(p \leq 0,05)$ con respecto a xilano y CMC, y similar entre estos dos últimos a las 3 (tabla I), y a las $6 \mathrm{~h}$ fue menor con CMC, pero a las $12 \mathrm{~h}$ se distinguen diferencias $(\mathrm{p} \leq 0,05)$ entre los sustratos ( sin sustrato $>$ xilano $>$ CMC). En la enzima con el NP 2 se presentaron menores promedios de $\mathrm{N}-\mathrm{NH}_{3}$ con respecto al 1; este efecto se mantuvo en todos los tiempos de incubación, además se observó una disminución de la concentración de $\mathrm{N}-\mathrm{NH}_{3}$ de las 3 a las 6 h en el NP 2. A las 12 h, en el NP 2, la concentración de N$\mathrm{NH}_{3}$ fue casi la mitad de la observada en el NP 1 . La interacción sustrato por NP fue significativa a las 6 y 12 h de incubación. En todos los tiempos de incubación las menores concentraciones de $\mathrm{N}-\mathrm{NH}_{3}$ se observaron en la enzima con xilano, CMC y sin sustrato en el NP 2. Para la enzima de $P$. ostreatus, la concentración de $\mathrm{N}-\mathrm{NH}_{3}$ fue diferente $(p \leq 0,05)$ entre enzima con xilano,

Archivos de zootecnia vol. 59, núm. 225, p. 146. 
DETERMINACIÓN DE LA DEGRADACIÓN DE LAS ENZIMAS EN LÍQUIDO RUMINAL

Tabla I. Concentración de $\mathrm{N}-\mathrm{NH}_{3}(\mathrm{mg} / \mathrm{dl})$ en líquido ruminal con enzimas de Trametes $\mathrm{sp}$. EUM1 o Pleurotus ostreatus IE8. (The $\mathrm{N}^{-\mathrm{NH}_{3}}(\mathrm{mg} / \mathrm{dl})$ concentration in ruminal liquid with enzymes of Trametes sp. EUM1 or Pleurotus ostreatus IE8).

\begin{tabular}{|c|c|c|c|c|c|c|}
\hline \multirow[b]{2}{*}{ Sustrato } & \multicolumn{3}{|c|}{$\begin{array}{c}\text { Trametes sp. } \\
\text { Tiempo de incubación (h) }\end{array}$} & \multicolumn{3}{|c|}{$\begin{array}{c}P . \text { ostretaus } \\
\text { Tiempo de incubación (h) }\end{array}$} \\
\hline & 3 & 6 & 12 & 3 & 6 & 12 \\
\hline Xilano & $3,24^{b}$ & $4,03^{a}$ & $5,64^{b}$ & $7,18^{c}$ & $7,69^{b}$ & $7,64^{\mathrm{b}}$ \\
\hline $\mathrm{CMC}$ & $2,62^{b}$ & $3,12^{\mathrm{b}}$ & $4,13^{c}$ & $9,72^{\mathrm{a}}$ & $9,90^{\mathrm{a}}$ & $10,33^{a}$ \\
\hline Sin sustrato & $6,51^{\mathrm{a}}$ & $4,40^{\mathrm{a}}$ & $7,29^{a}$ & $8,39^{b}$ & $9,97^{a}$ & $10,12^{\mathrm{a}}$ \\
\hline EEM & 0,506 & 0,253 & 0,410 & 0,290 & 0,234 & 0,315 \\
\hline \multicolumn{7}{|l|}{ NP } \\
\hline 1 & $4,13^{a}$ & $5,44^{a}$ & $7,38^{\mathrm{a}}$ & $8,92^{a}$ & 9,33 & $9,80^{\mathrm{a}}$ \\
\hline 2 & $2,90^{b}$ & $2,26^{b}$ & $3,99^{b}$ & $8,10^{\mathrm{b}}$ & 9,21 & $9,02^{b}$ \\
\hline EEM & 0,421 & 0,209 & 0,339 & 0,236 & 0,191 & 0,256 \\
\hline \multicolumn{7}{|l|}{ Sustrato*NP } \\
\hline Xilano-1 & 4,41 & $5,68^{a}$ & $7,42^{\mathrm{a}}$ & 7,60 & $7,30^{c}$ & 8,08 \\
\hline Xilano-2 & 2,08 & $2,37^{c}$ & $3,86^{c}$ & 6,76 & $8,08^{c}$ & 7,20 \\
\hline CMC-1 & 3,08 & $4,06^{b}$ & $5,03^{\mathrm{b}}$ & 10,19 & $10,45^{a}$ & 10,44 \\
\hline CMC-2 & 2,15 & $2,17^{c}$ & $3,22^{c}$ & 9,25 & $9,35^{\mathrm{b}}$ & 10,22 \\
\hline Sin sustrato-1 & 4,89 & $6,57^{a}$ & $9,70^{\mathrm{a}}$ & 9,28 & $10,23^{a}$ & 10,73 \\
\hline Sin sustrato-2 & 4,47 & $2,23 c$ & $4,88^{\mathrm{bc}}$ & 8,57 & $10,19^{a}$ & 9,51 \\
\hline EEM & 0,612 & 0,354 & 0,484 & 0,401 & 0,324 & 0,435 \\
\hline
\end{tabular}

$a, b, c$ Letras distintas en la misma fila en cada especie de hongo indican diferencias $(p<0,05)$.

$\mathrm{EEM}=$ error estándar de la media. $\mathrm{NP}=$ nivel de proteína. $\mathrm{CMC}=$ carboximetilcelulosa.

enzima con CMC y enzima sin sustrato a las 3 h, pero a las 6 y 12 h la concentración de $\mathrm{N}-\mathrm{NH}_{3}$ en la enzima con CMC y sin sustrato fue similar entre ellos y mayor con respecto a la enzima con xilano. Las concentraciones de $\mathrm{N}-\mathrm{NH}_{3}$ a las 3 y $12 \mathrm{~h}$ de incubación fueron mayores $(\mathrm{p} \leq 0,05)$ al NP 1 . Tanto en la enzima con CMC como en la enzima sin sustrato en el NP 1, la concentración de $\mathrm{N}-\mathrm{NH}_{3}$ fue mayor respecto al NP 2, pero con xilano fue menor en el NP 1. Los efectos del tiempo de contacto y el resto de las interacciones no fueron significativas en las enzimas de Trametes sp. y P. ostreatus. En la enzima de Fibrozyme, la concentración de $\mathrm{N}-\mathrm{NH}_{3}$ en enzima con xilano fue menor $(\mathrm{p} \leq 0,05)$ con respecto a la enzima con CMC y a la enzima sin sustrato en todos los tiempos de incubación (figura 1). No hubo efectos del NP, del tiempo de contacto ni de las interacciones.

La menor concentración de $\mathrm{N}-\mathrm{NH}_{3}$ que se detectó en el medio cuando se incluyó la enzima con sustrato, sugiere que la presencia de éste favoreció una menor degradación o le confirió mayor estabilidad a la enzima. Se indica que las enzimas que componen los productos comerciales usados como aditivos en alimentos para animales presentan estabilidad en el rumen (Hristov et al., 1998), atribuída en parte a la presencia de un sustrato, efecto asociado con la disminución de la sensibilidad de las enzimas (principalmente de las xilanasas) a la inactivación por proteinasas (Fontes et al., 1995). Aun cuando el análisis se hizo por separado para cada enzima, se observó que el tipo de sustrato puede ejercer efectos diferentes según el tipo de enzima, ya que la concentración de $\mathrm{N}-\mathrm{NH}_{3}$ en los tratamientos con enzimas de Trametes sp. fue mayor con xilano, mientras que para la enzima de $P$. ostretaus y Fibrozyme la mayor concentra- 


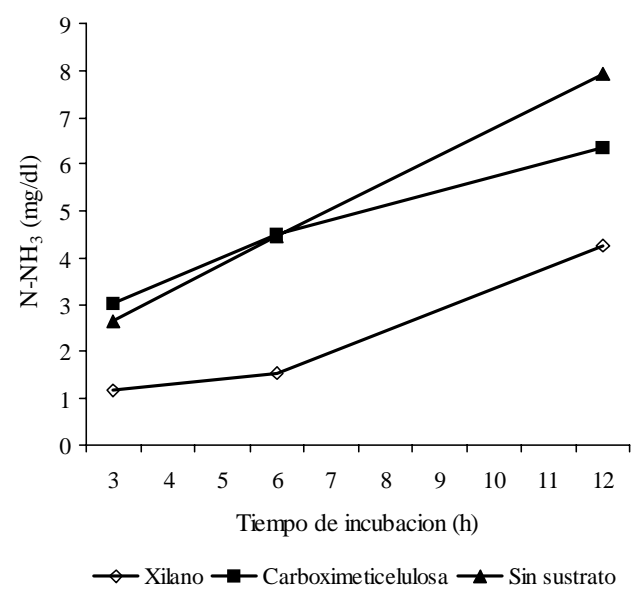

Figura 1. Concentración de $\mathrm{N}-\mathrm{NH}_{3}(\mathrm{mg} / \mathrm{dl})$ en líquido ruminal con Fibrozyme incubado con diferentes sustratos. (The $\mathrm{N}-\mathrm{NH}_{3}(\mathrm{mg} / \mathrm{dl})$ concentration in ruminal liquid with enzymes of Fibrozyme incubated with different substrates).

ción se obtuvo con el sustrato CMC.

En el mayor nivel de proteína de las

\section{BIBLIOGRAFÍA}

Bradford, M.M. 1976. Rapid and sensitive method for the quantization of microgram quantities of protein utilizing the principle of protein-dye binding. Anal. Biochem., 72: 248-252.

Dawson, K.A. and Tricarico, J.M. 1999. The use of exogenous fibrolytic enzymes to enhance microbial activities in the rumen and the performance on ruminant animals. In: Lyons, T.P. and K.A. Jacques (Eds.). Biotechnology in the Feed Industry. Proc. 15 $5^{\text {th }}$ Annu Symp. Nottingham University Press. pp. 303-312.

Fontes, C.M., Hall, J., Hirst, B.H., Hazlewood, G.P. and Gilbert, H.J. 1995. The resistance of cellulases and xylanases to proteolytic inactivation. Appl. Microbiol. Biotechol., 43: 52-57.

Hristov, A.N., McAllister, T.A. and Cheng, K-J. 1998. Stability of exogenous polysaccharidedegrading enzymes in the rumen. Anim. Feed Sci. Technol., 76: 161-168.

McAllister, T.A., Oosting, S.J., Popp, J.D., Mir, Z., Yake, L.J., Hristov, A.N., Treacher, R.J. and Cheng, K.J. 1999. Effect of exogenous enzymes enzimas de Trametes sp. y $P$. ostreatus se produjo menor concentración de $\mathrm{N}-\mathrm{NH}_{3}$, pero este efecto no se observó con Fibrozyme. A este respecto según Morgavi et al. (2001) una alta concentración de enzimas incrementa su estabilidad ante las proteasas del rumen con diferencias entre productos enzimáticos, lo que se atribuye al origen y a la estructura de las proteínas.

En conclusión, se observaron menores concentraciones de $\mathrm{N} . \mathrm{NH}_{3}$ al incubar enzimas con un sustrato, lo que indica que las enzimas de Trametes sp. EUM1, $P$. ostreatus IE8 y Fibrozyme en el líquido ruminal se degradaron menos en presencia de un sustrato, que podría considerse un factor importante en la estabilidad de las enzimas estudiadas. Los efectos fueron variables entre sustratos y tipos de enzima.

\section{AGRADECIMIENTOS}

A CONACYT (Proyecto No $42782-Z$ ) por la financiación parcial de la investigación.

on digestibility of barley silage and growth performance of feedlot cattle. Can. J. Anim. Sci., 79: 353-360.

McCoulloug, H. 1967. The determination of ammonia in whole blood by a direct colorimetric method. Clin. Chem. Acta., 17: 297-304.

Morgavi, D.P., Beauchemin, K.A., Nsereko, V.L., Rode, L.M., Iwaasa, A.D., Yang, W.Z., McAllister, T.A. and Wang, Y. 2000. Synergy between ruminal fibrolytic enzymes and enzymes from Trichoderma longibrachiatum. J. Dairy Sci., 83: 1310-1321.

Morgavi, D.P., Beauchemin, K.A., Nsereko, V.L., Rode, L., McAllister, T.A., Iwaasa, A.D., Wang, Y. and Yang, W.Z. 2001. Resistance of feed enzymes to proteolytic inactivation by rumen microorganism and gastrointestinal proteases. J. Anim. Sci., 79: 1621-1630.

SAS. 2001. SAS ${ }^{\circledR}$ Software, Version 8.2. SAS Institute, Inc.. Cary, NC. USA.

Steel, R.G. y Torrie, J.H. 1986. Bioestadística. Principios y procedimientos. (2a Ed.). McGrawHill Boock Co. México. 620 pp.

Archivos de zootecnia vol. 59, núm. 225, p. 148. 